\title{
O Uso das Tecnologias na Formação Inicial de Licenciandos em Ciências Biológicas: o que Dizem as Pesquisas na Área?
}

\author{
Micaelle G. Silva1, Dayane G. Silva1, Luiz G. Souza Neto1, Zélia M. Soares Jófiliı \\ 1Programa de Pós-Graduação em Ensino das Ciências (PPGEC) - Universidade Federal \\ Rural de Pernambuco (UFRPE) \\ Cep-52.171.900 - Recife - PE - Brazil \\ \{micaellegomes93,zjofili\}@gmail.com, \{Dayane.guimaraes2010, neto.hu\}@hot \\ mail.com
}

\begin{abstract}
Information and Communication Technologies (ICTs) have been increasingly expanding in the educational sphere, seeking to meet the constant needs of education. The present work had as objective to analyze the use of the technologies in the initial formation of Biology teachers. For this, a mapping of articles in periodicals and scientific events was carried out, covering the period from 2013 to 2017, and a total of 22 studies were identified on the use of technologies in the initial training of biology teachers. Despite the growing number of studies, it is still necessary to make progress in the use of technologies, to better subsidize teacher education.
\end{abstract}

Resumo. As Tecnologias da Informação e Comunicação (TIC) vêm se expandindo cada vez mais no âmbito educacional, buscando suprir as necessidades constantes da educação. O presente trabalho teve como objetivo analisar o uso das tecnologias na formação inicial de professores de Biologia e, para isto, foi realizado um mapeamento de artigos em periódicos e eventos científicos, abrangendo o período de 2013 a 2017. Foi identificado um total de 22 estudos abordando o uso de tecnologias na formação inicial de professores de biologia, mas apesar do crescente número de estudos, ainda se faz necessário avançar quanto ao uso das tecnologias, de modo a melhor subsidiar a formação docente.

\section{Introdução}

As instituições educacionais vêm demonstrando inquietações no que se refere ao ensino-aprendizagem de conteúdos específicos em qualquer área do conhecimento, sempre buscando a inclusão de estratégias didático-pedagógicas. No entanto, as lacunas persistem, fazendo-se necessário promover intervenções principalmente no que tange à base de conhecimento e formação docente.

Nesse cenário, as Tecnologias da Informação e Comunicação (TIC) vêm ganhando espaço em diversas áreas do conhecimento, sendo seu uso entendido como importante para apoiar o ensino. No entanto, [Gouvêa e Errobidart 2015] destacam que 
no contexto das atividades relacionadas ao ensino, a utilização das TIC ainda ocorre de forma limitada e ressaltam que os professores possuem a responsabilidade de avaliar os instrumentos pedagógicos de que fazem uso nas aulas, de modo a sanar as dificuldades no processo de ensino e aprendizagem.

Muitas dessas limitações encontram-se no processo de formação docente, onde é verificada a necessidade de estreitar relações entre teoria e prática nas aulas ministradas na licenciatura, unindo os conteúdos específicos, aos pedagógicos e tecnológicos. Isto implica acesso aos recursos, tempo suficiente para familiarização e adequação do material e suporte técnico, além é claro, da infraestrutura. Ou seja, a tecnologia por si só não significa solução e, mais do que isso, não funcionará para a melhoria da educação se não for utilizada com este propósito. Por isso, mudanças mais intensas devem ocorrer na formação docente, uma vez que são os professores, os responsáveis pela introdução dessas inovações no contexto do ensino.

[Bonzanini 2015] destaca a importância de promover, no decorrer da formação inicial, espaços para a reflexão crítica sobre o uso das TIC, de modo a possibilitar a inserção adequada das tecnologias no ensino dos conteúdos, propiciando, assim, uma prática pedagógica mais atual e dinâmica, que leve à construção dos novos conhecimentos.

[Oeiras et al. 2001] fazem menção a um saber específico recomendando que os professores, durante sua formação, sejam orientados a lançar mão dele no processo de ensino e aprendizagem de conteúdos específicos, garantindo não apenas um simples domínio instrumental, mas fornecendo subsídios para incorporar as TIC em suas aulas.

Desta forma, verifica-se a importância desses recursos na formação inicial de professores, nas distintas áreas do conhecimento. Neste estudo destacamos o Ensino de Biologia, tendo em vista as mais variadas formas de trabalhar conteúdos desta área por meio das tecnologias tão disseminadas atualmente.

Sendo assim, tecemos o estudo de forma a responder as seguintes questões de pesquisa: quais são as tecnologias educacionais utilizadas na formação inicial de licenciandos em Ciências Biológicas? Quais as contribuições dessas tecnologias na formação dos futuros professores de Ciências Biológicas? Em quais modalidades de ensino as tecnologias são trabalhadas?

Para tanto, o presente trabalho teve como objetivos analisar o uso das tecnologias na formação inicial de licenciandos em Ciências Biológicas, de modo a verificar as contribuições dessas tecnologias na formação dos futuros professores, bem como identificar os tipos de tecnologias mais utilizados nesse processo de formação inicial, como também em quais modalidades de ensino foram abordadas.

\section{As tecnologias na formação inicial de professores de Biologia}

A formação de professores é um tema central ao se pensar na qualidade do ensino, incluindo o de Biologia. Os docentes têm sido expostos a muitos desafios, como acompanhar as descobertas científicas e tecnológicas e torná-las compreensíveis para os alunos. Isto exige aprofundamento por parte do docente, tanto no campo do conhecimento teórico como no metodológico e tecnológico [Mishra e Koehler 2006]. Somado a isso, tais desafios podem ser agravados pelas deficiências nas licenciaturas, já que à medida que novas tecnologias surgem, assim como os conceitos se ampliam, a 
formação do professor passa a ser "obsoleta" poucos anos após a graduação dos mesmos [Lima e Vasconcelos 2006].

Nesse contexto, para auxiliar o docente nas questões de fazer com que os estudantes assimilem da melhor forma o que está sendo ensinado, as Tecnologias, se utilizadas de modo adequado, podem favorecer o processo de ensino e aprendizagem.

Nesse sentido, [Persich et al. 2015] destacam que no contexto da formação inicial dos licenciandos em Ciências Biológicas, se faz necessária a percepção da importância das TIC como ferramentas e recursos didáticos aliados do processo da aprendizagem e do ensino.

Sendo assim, se faz necessária a inclusão das TIC como ferramentas didáticas importantes na construção do conhecimento pelos alunos das licenciaturas, em específico da área da Biologia para que possa auxiliar tanto no processo de aprendizagem durante a graduação, como no processo de ensino, para utilização efetiva desses meios tecnológicos nos ambientes escolares.

$\mathrm{Na}$ tentativa de verificar o que está sendo investigado no campo do Ensino das Ciências, sobre a utilização das tecnologias na formação inicial de licenciandos em Ciências Biológicas, apresentamos, neste trabalho, um levantamento de pesquisas com esta abordagem em eventos e periódicos nacionais e internacionais.

Vale destacar a expansão da produção científica acerca das tecnologia nas áreas de ensino, seja no âmbito da Educação Básica, seja no Ensino Superior. No Ensino de Biologia, estas têm tomando uma dimensão bastante significativa. De acordo com [Oliveira 2015] aspectos biológicos são tidos como complexos e aprender Biologia é muito mais do que decorar conceitos, é também reconhecer processos que ocorrem na natureza. Nesse sentido, [Gianotto e Diniz 2010] ressaltam a utilização da tecnologia para uma aprendizagem eficaz no que se refere ao Ensino de Biologia de modo a desmitificar os conteúdos tidos como complexos.

Mediante a disseminação dos meios tecnológicos na contemporaneidade e por verificarmos nos canais de divulgação de trabalhos científicos, artigos sobre o uso das tecnologias no Ensino de Biologia para a Educação Básica, decidimos investigar o uso dessas tecnologias na formação inicial de licenciandos em Ciências Biológicas, tendo em vista que para ser utilizados no Ensino de Biologia no campo da Educação Básica, de forma a contribuir na aprendizagem dos estudantes é relevante a abordagem dessas ferramentas na formação inicial desses profissionais.

\section{Caminhar Metodológico}

O presente estudo caracteriza-se por uma abordagem de cunho quanti-qualitativa [Minayo 2011], e investiga o estado da arte das produções cientificas de artigos, em determinado assunto, considerando: tempo, espaço e mecanismo de publicação [Ferreira 2002]. Foi realizado um mapeamento em publicações no período temporal de 2013 a 2017, pois demarcamos um período de cinco anos para está análise, em um evento e em revistas nacionais e internacionais do seguimento de ensino.

O corpus da análise da pesquisa é constituído por artigos do principal evento nacional de Ensino de Ciências Brasileiro e de quatro revistas nacionais e internacionais de Qualis A1 e A2 na área de Ensino, de acordo com a última avaliação quadrienal (2013-2016). A seleção das revistas analisadas se deu pelo perfil de publicação das mesmas e o objeto da investigação da pesquisa, conforme a lista a seguir: Evento - 
Encontro Nacional de Pesquisa em Educação em Ciências (ENPEC) dos anos de 2013, 2015 e 2017; Revistas Nacionais - Revista Brasileira de Ensino de Ciência e Tecnologia (RBECT) - Qualis A2 de 2013 a 2017 e Investigações em Ensino de Ciências (IEC) Qualis A2 - de 2013 a 2017; e Revistas Internacionais - Ensenãnza de Las Ciencias Qualis A1 dos anos de 2013 e 2017 e Revista Latinoamericana de Tecnología Educativa (RE) - Qualis A2 de 2013 a 2017.

A busca dos artigos foi feita através de sites. No caso do único evento, foi a partir de sua promotora que é a Associação Brasileira de Pesquisa em Educação em Ciências (ABRAPEC) que nos subsidiou o link de acesso aos anais do evento. No caso das revistas nacionais e internacionais os artigos foram localizados a partir dos sites de busca. Para a seleção inicial dos artigos realizamos uma identificação prévia dos textos utilizando, como meio de filtragem: título, resumo, palavras-chave (buscando pelos termos "Formação Inicial", "Licenciandos", "Ensino de Biologia", "Tecnologia" e "TIC". A exclusão de artigos não condizentes com a temática, embora houvesse alguma das palavras destacadas nos títulos, resumos e palavras-chaves, ocorreu no momento da exploração do material, na leitura aprofundada destacado a seguir.

Logo após esse momento nos guiamos e seguimos para a análise dos corpus coletados seguindo alguns passos da Análise de Conteúdo de [Bardin 2011]. Desta forma, ocorreram os respectivos polos cronológicos indicados pela autora: Pré-análise leitura flutuante e construção dos indicadores; Exploração do material - leitura aprofundada e categorização e tratamento dos resultados, inferência e interpretação. Ressaltamos que o agrupamento e, como resultado, as categorizações, ocorreram em um momento a posteriori, partindo das tendências que identificamos de uso das tecnologias na formação inicial de licenciandos em Ciências Biológicas.

\section{Resultados e Discussões}

A partir das técnicas anteriormente mencionadas foi possível coletar e montar a seguinte amostra do corpus do estudo e, consequentemente, montar um quadro dos artigos e suas fontes de publicações, conforme demonstrado no Quadro 1. Tal resultado expõe como determinada temática está sendo publicada na área de ensino no período temporal de 2013-2017 no seguimento de práticas e teorias da formação inicial de licenciandos em Ciências Biológicas correlacionados com as tecnologias.

De acordo com os termos de busca especificados para a pesquisa foram retornados 24 artigos científicos, contudo no momento da exploração dos achados, com uma leitura aprofundada, dois artigos foram excluídos, pois embora os títulos apresentassem termos destinados para seleção dos artigos o conteúdo apresentado no corpo do artigo não era condizente com os objetivos traçados nesta pesquisa.

Quadro 1. Distribuição e quantitativo dos artigos por Evento, Revistas Nacionais e Internacionais nos anos investigados.

\begin{tabular}{|l|l|c|c|c|c|c|c|}
\hline \multirow{2}{*}{$\begin{array}{c}\text { Fontes das } \\
\text { publicações }\end{array}$} & Evento e Revistas & \multicolumn{5}{|c|}{ Anos } & $\begin{array}{c}\text { Total de artigos por } \\
\text { Evento e Revistas }\end{array}$ \\
\cline { 2 - 8 } & & $\mathbf{2 0 1 3}$ & $\mathbf{2 0 1 4}$ & $\mathbf{2 0 1 5}$ & $\mathbf{2 0 1 6}$ & $\mathbf{2 0 1 7}$ & 13 \\
\hline Evento & ENPEC & 5 & - & 4 & - & 4 & 5 \\
\hline Revistas Nacionais & RBECT & 1 & 3 & 0 & 0 & 1 & 0 \\
\cline { 2 - 8 } & IEC & 0 & 0 & 0 & 0 & 0 & 4 \\
\hline $\begin{array}{l}\text { Revistas } \\
\text { Internacionais }\end{array}$ & $\begin{array}{l}\text { Ensenãnza de Las } \\
\text { Ciencias }\end{array}$ & 2 & - & - & - & 2 & 0 \\
\cline { 2 - 7 } & RELATEC & 0 & 0 & 0 & 0 & 0 & \\
\hline
\end{tabular}


Dentre a amostra selecionada, detectamos e exemplificamos um maior número de artigos sobre a temática em estudo no ENPEC, em suas três edições no período investigado, totalizando treze dos 22 artigos. Ressaltamos que tal dado deve-se ao fato do maior quantitativo de artigos que um evento aceita por edição, em relação às revistas. Posteriormente, conseguimos coletar quatro dos demais artigos na Revista Internacional "Ensenãnza de Las Ciencias" resultante das duas últimas edições do "Congreso Internacional sobre Investigación en Didáctica de las Ciencias" que aconteceram respectivamente nas cidades de Girona e Sevilha, na Espanha, com quatro artigos e também na Revista Nacional RBECT onde, em três anos, dos cinco investigados, foram obtidos cinco artigos, totalizando assim nove artigos nas duas revistas. Não detectamos artigos da temática sob estudo nem na Revista Nacional IENCI nem na Revista Internacional RELATEC, no período investigado.

Durante a análise qualitativa dos corpus empíricos dos artigos supracitados, conseguimos elencar duas categorias de interesse da pesquisa: A. Modalidade de ensino e B. Tipos de tecnologias.

A. Modalidade de Ensino (Presencial, Semipresencial e Educação a Distância EAD): A presente categoria parte da perspectiva de que na formação de licenciandos em ciências biológicas no ensino superior, existem diferentes tipos de modalidades de ensino tanto em instituições públicas federais e estaduais como em instituições particulares. Por conseguinte, na análise detectamos um número relevante de artigos na modalidade EAD, o que nos pareceu importante para ser discutido na pesquisa.

B. Tipos de Tecnologias: Nesta categoria detectamos o uso de diferentes recursos tecnológicos utilizados na formação dos licenciandos, o que também nos pareceu importante para ser discutido na pesquisa.

No que se refere ao foco do estudo verificamos um predomínio da modalidade presencial de ensino com treze artigos: [Bastos, Rezende Filho e Pastor 2013] e [Corrêa e Caldeira 2017]. Tal resultado já era esperado, tendo em vista a quantidade de cursos na modalidade presencial na formação dos licenciandos. Seguidamente, a modalidade EAD apresentou cinco artigos com discussões relevantes acerca dos desafios, potencialidades e reflexões do papel didático do ambiente virtual sobre a tecnologia já incorporada na formação dos licenciandos como o ambiente hipermídia [Espíndola Santos e Silva 2013] [André 2014].

Os artigos de [Costa e Rezende 2013] e [Silva, Ribeiro e Gastal 2013] tiveram como viés de interesse um olhar sobre os licenciandos oriundos da Educação à Distância, tanto para verificar à reflexão na formação inicial quanto na dos futuros profissionais e a construção da identidades destes licenciandos. Cabe ressaltar que essa modalidade de ensino foi regulamentada desde 1996 com a Lei de Diretrizes e Bases $n^{\circ}$ 9.394/96, tendo então mais de duas décadas de existência.

Para a modalidade semipresencial encontramos e analisamos dois artigos. O de [Bonzanini 2015] que apresentou uma reflexão sobre a formação pedagógica para o uso das tecnologias em licenciandos de diferentes anos de turmas semipresenciais; e o de [Maganha et al. 2017] que teve como objetivo compreender como os tutores mediavam os fóruns de uma disciplina e realizavam perguntas em turmas em determinado período de tempo, também na modalidade semipresencial. 
Por fim, salientamos que foram analisados dois artigos que não exemplificaram nenhuma modalidade de ensino, tendo em vista, serem pesquisas bibliográficas. $\mathrm{O}$ estudo de [Silva et al. 2014] teve o objetivo de investigar o perfil tecnológico dos licenciandos de ciências biológicas e o artigo de [Possas e Alves 2015] fez uma revisão bibliográfica com o objetivo de responder questionamentos sobre o uso das TIC no ensino de biologia no geral, enfocando a formação dos licenciandos.

No que se refere ao subtópico Tipos de Tecnologia, buscamos investigar o corpus dos artigos com a preocupação de identificar as tecnologias que foram empregadas nas publicações acadêmicas. A partir da amostra do estudo notamos que as tecnologias foram empregadas e citadas como uma importante ferramenta de auxílio durante o momento de ensino e aprendizagem dos licenciandos, conforme o trecho do artigo de [Pereira e Lopes 2015] que foi um artigo de viés teórico,

\section{(...) as TIC influenciam diretamente no processo de formação do aluno, pois quando usadas como ferramentas no processo de aprendizagem, contextualizam o educando na sociedade em que está inserido, tornando-o um cidadão crítico e sujeito do seu processo de formação [p.4].}

Outro olhar que constatamos nos artigos foi a utilização do Ambiente Virtual de Aprendizagem (AVA) partindo do uso da hipermídia no ensino dos licenciandos, apontadas nas potencialidades que os professores trazem no estudo de [Espíndola Santos e Silva 2013] Pela fala dos professores, percebe-se que a hipermídia cumpriu um aspecto predominantemente "facilitador" da compreensão e como meio de "despertar o interesse dos alunos"

A hipermídia contribuiu de maneira positiva para o aprendizado dos alunos, que tiveram a oportunidade de estudar o tema de maneira dinâmica, divertida, interessante e didática, uma alternativa importante de ferramenta de estudos [Espíndola Santos e Silva 2013].

Esta perspectiva também foi discutida por [André 2014] partindo de reflexões da Educação à Distância e do AVA, no que diz respeito à função e ao papel dos docentes no ambiente hipermídia, tendo que se aprimorar para propiciar melhores momentos de ensino e aprendizagem nas suas aulas. Outro aspecto relevante foi observado no estudo de [Montenegro Petrovich e Araújo 2013] quando trazem um relato de experiência de licenciandos, alunos da Educação à distância, que descreve uma oficina presencial na Universidade Federal do Rio Grande do Norte com conteúdos da zoologia em que utilizaram tanto modelos didáticos de biscuit quanto recursos tecnológicos, como computadores, apresentação de slides e apresentação de vídeos, apontando assim, a possível relação entre o uso de aulas práticas, materiais didáticos e recursos tecnológicos na formação inicial de licenciandos da Educação à Distância.

Verificamos também a reprodução de vídeos, produção de vídeo didático e análise de vídeos como funcionalidades dadas a este recurso tecnológico recorrente nos relatos dos artigos analisados. Por fim, trazemos três outras funções observadas em três artigos distintos: primeiramente o texto de [Maganha et al. 2017] quando analisam a condução de tutores em atividades assíncronas, por meio de fóruns. Em seguida, trazemos o artigo de [Eichler e Del Pino 2014] no qual relatam o uso do software Jigo que é um editor de criação que possibilita a construção de objetos de aprendizagem, onde é possível planejar paisagens, ambientes e espaços naturais.

A última função destacada foi o relato de [Corrêa e Caldeira 2017] quando, em uma disciplina pedagógica da licenciatura, solicitam que duas turmas de licenciandos elaborem atividades didáticas mediadas por TIC, onde foram propostos e indicados os 
seguintes recursos: (1) As redes sociais como ferramenta de ensino; 2) Ensinando por meio de jogos digitais; 3) Giz e lousa versus computadores e datashow na sala de aula. Dessa forma foi possibilitado um planejamento pedagógico correlacionado com recursos tecnológicos, tornando possível um questionamento dos licenciandos ainda na formação inicial.

Apesar dos trabalhos encontrados estarem direcionados para as três modalidades de ensino (presencial, semipresencial e educação à distância) na formação de professores, assim como diferentes tipos de tecnologias utilizados nesses artigos. $\mathrm{O}$ número de produções destinadas a essa temática no processo de formação inicial de professores de ciências biológicas, ainda se encontra incipiente. Um dos aspectos que podem ser citados se relaciona ao fato da aproximação dos docentes formadores com esses tipos de tecnologias, podendo nos cursos de formação continuada de professores ser promovida formações para utilização da tecnologias no processo de ensino e aprendizagem.

\section{Considerações Finais}

Os trabalhos analisados destacaram o uso das tecnologias na formação inicial dos licenciandos em diferentes modalidades de ensino como Educação à distância, semipresencial e presencial, chamando a atenção para o números de pesquisas nos Cursos de Educação à Distância (EAD) que foi a segunda mais abordada uma vez que a EAD vem se expandindo bastante nos últimos anos.

Em suma, as pesquisas aqui analisadas demonstraram um direcionamento do uso das tecnologias e suas implicações para o processo de ensino e aprendizagem dos discentes dos cursos de Ciências Biológicas em formação inicial, bem como as contribuições desses meios tecnológicos na futura prática docente. Contudo, ainda se faz necessário avançar nas pesquisas quanto ao uso das tecnologias na formação inicial de professores de Ciências Biológicas, de modo a oferecer um panorama acerca do que está sendo estudado nesta perspectiva e promover mais subsídios para a formação docente.

\section{Referências}

André, B.P. (2014). O lugar da didática no ambiente virtual de aprendizagem. Revista Brasileira de Ensino de Ciência e Tecnologia, v. 7, n. 3, p. 63-77.

Bastos, W.G.; Rezende Filho, L.A.C.; Pastor, A.A. (2013). Produção de vídeos educativos por licenciandos de Biologia: uma análise do endereçamento e do significado preferencial. In: Encontro Nacional de Pesquisa em Educação em Ciências, São Paulo, Atas do IX ENPEC Encontro Nacional de Pesquisa em Educação em Ciências. São Paulo: ABRAPEC.

Bardin, L. (2011). Primeira parte - história e Teoria; Terceira parte - Método. Análise de Conteúdo. Lisboa: Editora 70.

Bonzanini, T.K. (2015). A formação do professor para o uso pedagógico das tecnologias: investigações em um curso de licenciatura em ciências. In: Encontro Nacional de Pesquisa em Educação em Ciências, Águas de Lindóia, Atas do X ENPEC Encontro Nacional de Pesquisa em Educação em Ciências. São Paulo: ABRAPEC. 
Corrêa, A.L.; Caldeira, A.M.A. (2017). Proposta de competências necessárias para o ensino de Ciências e Biologia em atividades mediadas por TIC. In: Encontro Nacional de Pesquisa em Educação em Ciências, Florianópolis, Atas do XI ENPEC Encontro Nacional de Pesquisa em Educação em Ciências. Santa Catarina: ABRAPEC.

Costa, M.L.R.; Rezende, F. (2013). Construção da identidade docente em um curso a distância de Licenciatura em Biologia. In: Encontro Nacional de Pesquisa em Educação em Ciências, São Paulo, Atas do IX ENPEC Encontro Nacional de Pesquisa em Educação em Ciências. São Paulo: ABRAPEC.

Eichler, M.L.; Del Pino, J.C. (2014). Jigo: um editor de objetos de aprendizagem de temas de Ciência, Tecnologia e Sociedade (CTS). Revista Brasileira de Ensino de Ciência e Tecnologia, v. 7, n. 1, p. 119-141.

Espíndola, M.B.; Santos, J.V.A.; Silva, C.F. (2013). Análise do processo de desenvolvimento e uso de uma hipermídia no ensino superior de Ciências Biológicas. In: Encontro Nacional de Pesquisa em Educação em Ciências, São Paulo, Atas do IX ENPEC Encontro Nacional de Pesquisa em Educação em Ciências. São Paulo: ABRAPEC.

Ferreira, N.S.A. (2002) As pesquisas denominadas "estado da arte". Educação \& Sociedade, n. 79, p. 257-272.

Gianotto, D.E.P.; Diniz, R.E.S. (2010). Formação inicial de professores de Biologia: a metodologia colaborativa mediada pelo computador e a aprendizagem para a docência. Ciência \& Educação, Bauru, v. 16, n. 3, p. 631-648.

Gouvêa, S.M.O.; Errobidart, N.C.G. (2015). A formação de professores para novas tecnologias: reflexões a partir da literatura. In: X Encontro Nacional de Pesquisa em Educação em Ciências, Águas de Lindóia, Atas do X ENPEC- Encontro Nacional de Pesquisa em Educação em Ciências. Águas de Lindóia: ABRABEC.

Lima, K.E.C.; Vasconcelos, S.D. (2006). Análise da metodologia de ensino de ciências nas escolas da rede municipal de Recife. Ensaio: Avaliação e Políticas Públicas em Educação. Rio de Janeiro, v.14, n.52, p. 397-412.

Maganha, L.F. et al. (2017). O papel do tutor em fóruns de discussão em um curso semipresencial de Licenciatura em Ciências: A importância do tipo de pergunta. In: Encontro Nacional de Pesquisa em Educação em Ciências, Florianópolis, Atas do XI ENPEC Encontro Nacional de Pesquisa em Educação em Ciências. Santa Catarina: ABRAPEC.

Minayo, M.C.S. (2011). Pesquisa social: teoria, método e criatividade. Rio de Janeiro: Editora Vozes Limitada.

Mishra, P.; Koehler, M. (2006). Technological pedagogical content knowledge: a new framework for teacher knowledge. Teacher College Record, v. 108, n. 6, p. 10171054.

Montenegro, L.A.; Petrovich, A.C.I.; Araújo, M.F.F. (2013). Modelos biológicos no estudo de vertebrados: utilização e importância na formação inicial à distância de professores para o ensino de ciências e biologia. In: Encontro Nacional de Pesquisa em Educação em Ciências, São Paulo, Atas do IX ENPEC Encontro Nacional de Pesquisa em Educação em Ciências. São Paulo: ABRAPEC. 
Oeiras, J.Y.Y. et al. (2001). Contribuições de conceitos de Comunicação Mediada por Computadores e visualização de informação para o desenvolvimento de ambientes de aprendizagem colaborativa. In: XII SIMPÓSIO BRASILEIRO DE INFORMÁTICA NA EDUCAÇÃO, SBC, Vitória do Espírito Santo. Espírito Santo: Anais do Simpósio.

Oliveira, B.I.N. (2015). Tecnologias aplicadas ao ensino de biologia: o uso dos tablets em escolas estaduais do município de patos - PB. 34f. Trabalho de Conclusão de Curso (Licenciatura em Ciências Biológicas). Universidade Federal de Campina Grande.

Persich, G.D.O. (2015). O uso de Tecnologias de Informação e Comunicação (TIC) por participantes do PIBID Ciências Biológicas em Santo Ângelo (RS) e Ciências da Natureza em Senhor do Bonfim (BA). In: X Encontro Nacional de Pesquisa em Educação em Ciências, Águas de Lindóia, Atas do X ENPEC Encontro Nacional de Pesquisa em Educação em Ciências. São Paulo: ABRAPEC.

Pereira, M.; Lopes, L.A. (2015). Tecnologias de Informação e Comunicação na Educação: horizontes para a formação de professores de Ciências. In: Encontro Nacional de Pesquisa em Educação em Ciências, Águas de Lindóia, Atas do X ENPEC Encontro Nacional de Pesquisa em Educação em Ciências. São Paulo: ABRAPEC.

Possas, I.M.M.; Alves, J.M. (2015). Estudo exploratório das pesquisas sobre TIC no ensino de Biologia. In: Encontro Nacional de Pesquisa em Educação em Ciências, Águas de Lindóia, Atas do X ENPEC Encontro Nacional de Pesquisa em Educação em Ciências. São Paulo: ABRAPEC.

Silva, S.L.R. et al. (2014). Perfil tecnológico de acadêmicos de cursos de licenciatura das ciências da natureza. Revista Brasileira de Ensino de Ciência e Tecnologia, v. 7, n. 3, p. 135-147.

Silva, C.M.; Ribeiro, A.M.; Gastal, M.L.A. (2013). Formação de professores de Ciências: refletindo sobre a prática docente através do estágio supervisionado em um curso de licenciatura à distância em biologia. In: Encontro Nacional de Pesquisa em Educação em Ciências, São Paulo, Atas do IX ENPEC Encontro Nacional de Pesquisa em Educação em Ciências. São Paulo: ABRAPEC. 